\title{
Ultra-compact SWIR telephoto lens design with SMS method
}

\author{
Wang Lin ${ }^{1}$, Pablo Benítez ${ }^{1,2}$, J.C.Miñano ${ }^{1,2}$,Jose Infante ${ }^{1,3}$, \\ Marta de la fuente ${ }^{3}$, Guillermo Biot ${ }^{1}$ \\ 1. CEDINT, Universidad Politécnica de Madrid \\ 2. LPI-LLC, USA \\ 3. Indra Sistemas SA, Spain
}

\begin{abstract}
:
In this work, we propose two new optical structures, using the Simultaneous Multiple Surfaces (SMS) method, comprised of 2 reflecting surfaces and 2 refracting surfaces, $800 \mathrm{~mm}$ focal length, f/8 (aperture diameter $100 \mathrm{~mm}$ ) and $1.18^{\circ}$ diagonal field of view in the SWIR band. The lens surfaces are rotational symmetric and calculated to have good control of non-paraxial rays. We have achieved designs with excellent performance, and with total system length of less than $60 \mathrm{~mm}$.
\end{abstract}

KEYWORDS

SMS method, telephoto lens, SWIR, imaging system

\section{Introduction}

Recently, with the development of InGaAs sensors, the short-wave infrared (SWIR) band (from 0.9um to 1.7um) has been found to have wide applications in machine vision and military fields of use ${ }^{[1,2,3,4,5]}$. Light within this band cannot be seen by human eyes, but interacts with objects in a similar way as visible wavelengths. Therefore, it can be used to produce images similar to visible images in resolution and detail. In this work, we shall design a SWIR tele-objective system with $800 \mathrm{~mm}$ focal length and $1.18^{0}$ diagonal field of view. The difficulty in these Catadioptric-type designs is to maintain a high performance with small overall system length, which is very important in space-limited applications, such as a UAV surveillance system.

In order to provide a good initial starting point for further optimization, the SMS2D (Simultaneous Multiple Surfaces in 2 dimensions) method has been used to construct an initial design. The SMS method was developed primarily as a design method in Nonimaging Optics during the $1990 \mathrm{~s}^{[6-8]}$. Later, the method was extended for designing Imaging Optics. The application of the SMS method for imaging optics was first introduced for the design of an ultra-high numerical aperture RX lens composed by two aspheric surfaces ${ }^{[9]}$. More recently, the extension to up to four aspherics was developed ${ }^{[10-12]}$. Different from traditional optical design methods based on multi-parametric optimization techniques, the SMS method is a direct construction method for selected ray-bundles, meridian or skewed ray-bundles ${ }^{[13,14]}$. This gives the SMS method an additional interest since it can be used for exploring solutions far from the paraxial approximation where the multi-parametric techniques can get lost because of the multiple local minima.

\section{SMS design processes}

The standard SMS2D process includes two parts: Initial curves selection and Recursive Cartesian oval calculation ${ }^{[9-14]}$. The initial curves selection plays an important role in the SMS calculation because all the curves will grow in small segments from selected initial curves. A good selection of initial curves differs greatly from a bad selection in both stability of the SMS process and performance of the final optical design. Since the central vertices of initial 
curves can be defined loosely from mechanical or optical requirements of the system, a set of criteria is need for selection of central shape of initial curves. From paraxial image calculation or aplanatic conditions, initial curves can only control axial rays, without considering non-axial rays.

Here we propose a set of wavefront criteria for 3 initial curves selection, as shown in Fig 1. The theory of the SMS method states that 3 surfaces can perfectly image 3 input wavefronts into 3 output wavefronts ${ }^{[8]}$. In this work, only meridian ray bundles are selected for SMS2D design, therefore they are placed symmetrically about the optical axis, which is a reasonable representation of all the meridian ray bundles. Points $V_{1}, V_{2}, V_{3}$ are central vertices of the initial curves, that can be defined beforehand by system requirements. $R_{2}$ is the chief ray which passes from an on-axis point $\mathrm{Obj}_{2}$ to an on-axis point $\mathrm{Img}_{2}$ along the optical axis. $\mathrm{R}_{1}$ is the chief ray which passes from an off-axis point $\mathrm{Obj}_{1}$ to an off-axis point $\mathrm{Img}_{1}$ and $\mathrm{R}_{3}$ is its symmetrical counterpart. $\mathrm{R}_{1}, \mathrm{R}_{2}, \mathrm{R}_{3}$ all pass through the vertex of middle curve: $\mathrm{V}_{2}$.

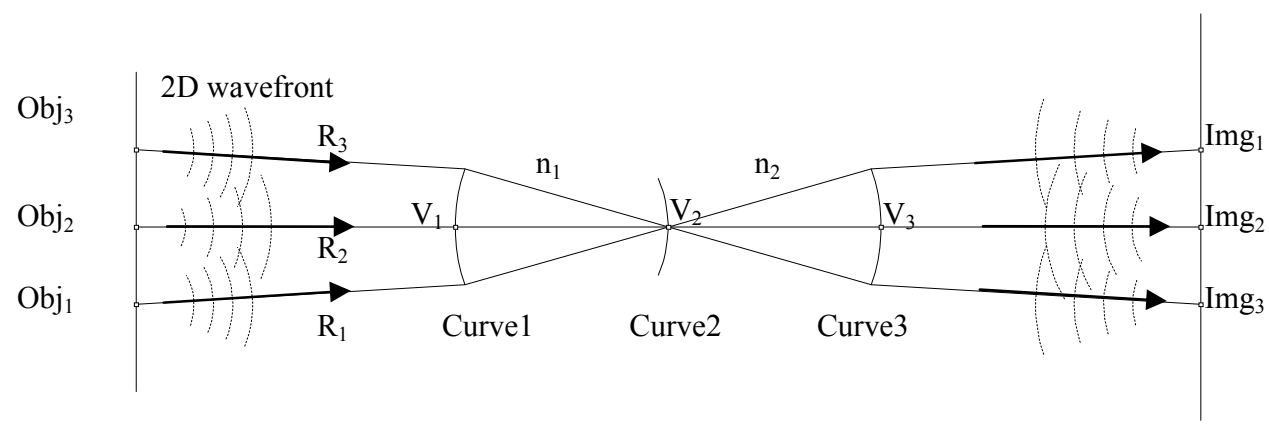

Fig 1. Wavefront criteria for initial curves selection
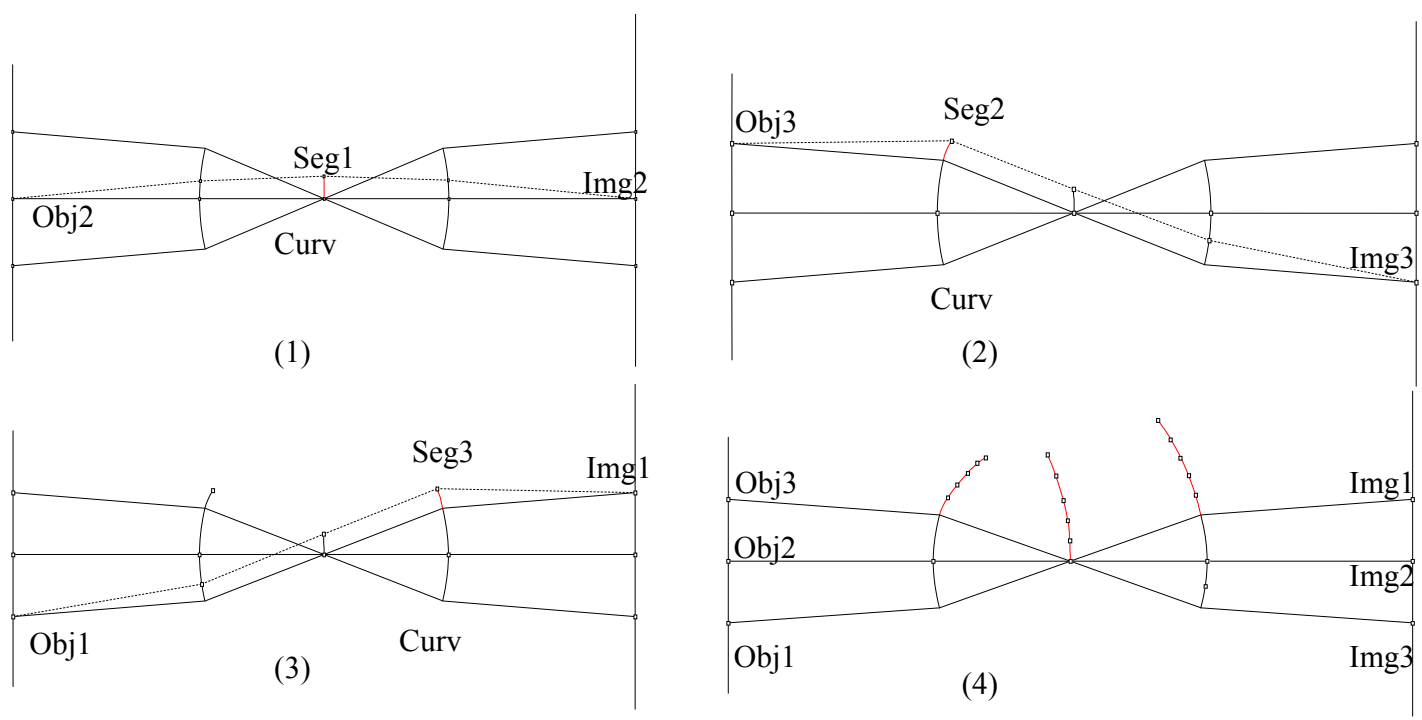

Fig 2. SMS growth process

The wavefront criteria requires that: the meridian wavefronts around the chief rays of 3 object points should be transformed into the meridian wavefronts of their corresponding image points by passing through this rotational optical system with generalized ray-tracing ${ }^{[15]}$. In this way, not only axial rays, but also non-axial meridian rays 
around $R_{1}$ and $R_{3}$ form perfect images by initial central curves. The solution to wavefront criteria might not be unique. It often results in several different shapes of initial curves. Later, after an SMS construction for each selection of initial curves, it can be determined which is the better solution, by simply raytracing designed ray-bundles (meridian ray-bundles in our case). The better solution to wavefront criteria should have better control of the designed rays.

Once the initial curves are selected, the rest part of each curve can be grown from the initial curves, as shown in Fig 2. Primarily, a small segment Seg1 of curve 2 is calculated as a Cartesian oval from the existing part of curve 1 and curve 3 to image $\mathrm{Obj}_{2}$ into $\mathrm{Img}_{2}$; secondarily, a small segment Seg2 of curve 1 is then calculated through Seg1 and curve 3 to image $\mathrm{Obj}_{3}$ into $\mathrm{Img}_{3}$; tertiarily, Seg3 of curve 3 can be calculated from Seg1 and curve 1 to image $\mathrm{Obj}_{1}$ into $\operatorname{Img}_{1}$. By repeating the same process, all segments can then be grown from the initial curves.

\section{Tele-photo design}

In SWIR band (0.9um-1.7um), glass properties like abbe value are generally different from those in the visible band, and number of possible crown-flint combinations are also reduced, compared to the visible band ${ }^{[4,5]}$. Many crown materials in the visible band can change to flint materials in the SWIR band. Therefore, we will start with a monochromatic design for the $1.3 \mathrm{um}$ wavelength, using an artificial material with refractive index 1.5 . Optical parameters are listed in Table 1.

Table 1. optical parameters

\begin{tabular}{|l|l|}
\hline Wavelength & $0.9 \mathrm{um}-1.7 \mathrm{um}$ \\
\hline Focal length & $800 \mathrm{~mm}$ \\
\hline F\# & 8 \\
\hline Field of view & $1.18^{0}$ \\
\hline Refractive index & $1.5($ artificial material) \\
\hline Optical dimensions & $100 \mathrm{~mm}$ diameter \\
& $60 \mathrm{~mm}$ system length \\
\hline
\end{tabular}

In order to achieve high compactness with high optical performance, two optical structures are proposed as candidates for the tele-objective design: named "RXXR" and "XRXR". In our nomenclature: "R" refers to a refractive surface, and " $X$ " refers to a reflective surface. Two structures are named from object space to image space by orders of the surfaces' types, shown in Fig 3. 


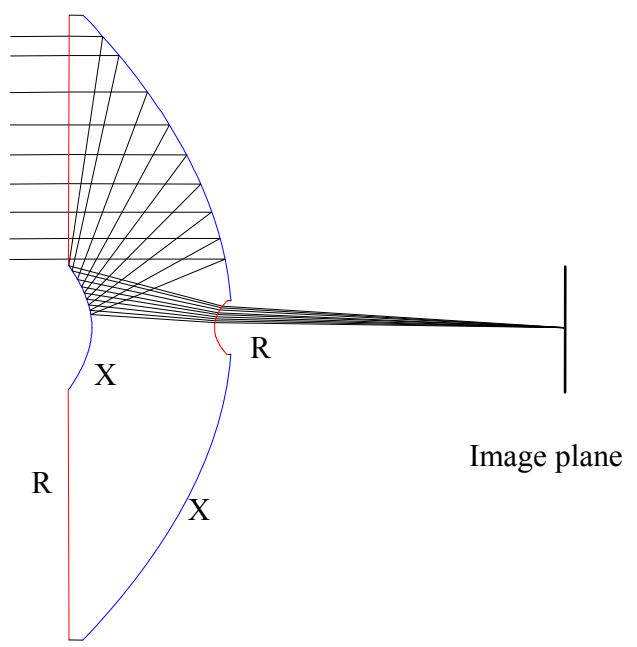

Type 1: RXXR

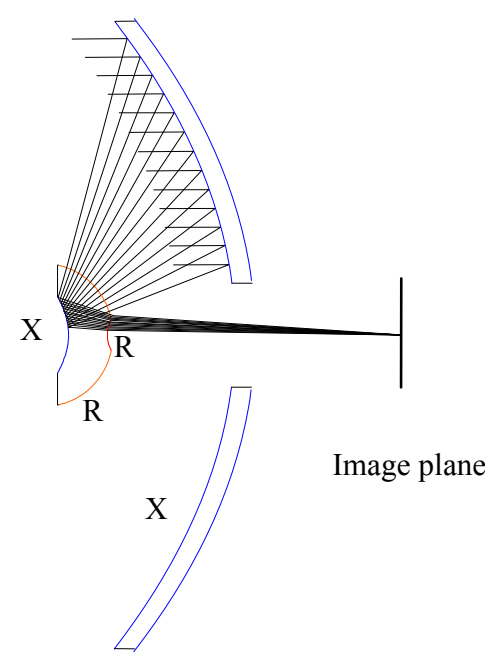

Type 2: XRXR

Fig 3. sketches of 2 candidate structures

Comparing these two structures, it can be seen that:

a. Type RXXR can be made into one solid lens with two metalized surfaces, and type XRXR can be made into two pieces( one mirror and one lens). Therefore, type RXXR seems to be more compact than type XRXR.

b. Considering the weight of the whole system, type RXXR uses more volume of material than type XRXR. Therefore, type RXXR seems to weigh more than type XRXR.

In both structures, there are 4 surfaces to be designed. In order to use the algorithm described in section 3, 1 surface should be prescribed beforehand, so that other 3 surfaces can then be grown with the SMS2D method. In a type RXXR, the first refractive surface is pre-given as a parabola with a fixed center position and close to 0 central curvature; in type XRXR, the first reflective surface is also pre-given as a parabola with a fixed center position and a certain curvature. Because the design procedures for type RXXR and XRXR are very similar, we will only present the process for type RXXR.

By applying the wavefront criteria to the initial curves, two solutions are found for the same given first surface, shown in Fig 4. 


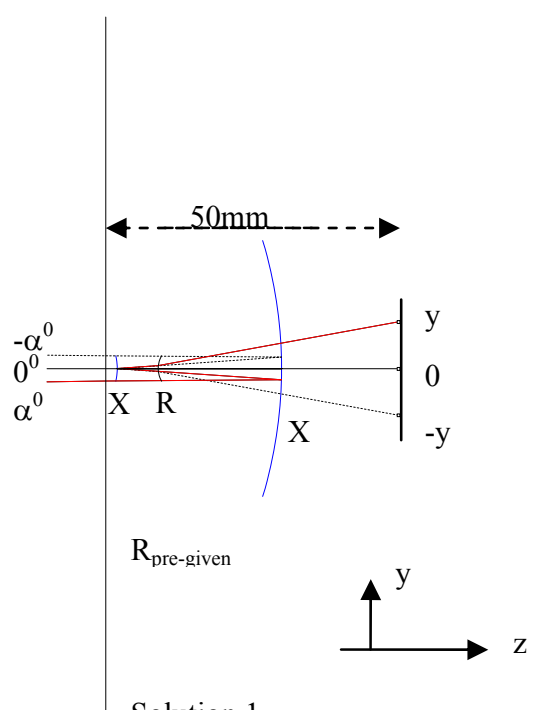

Solution 1

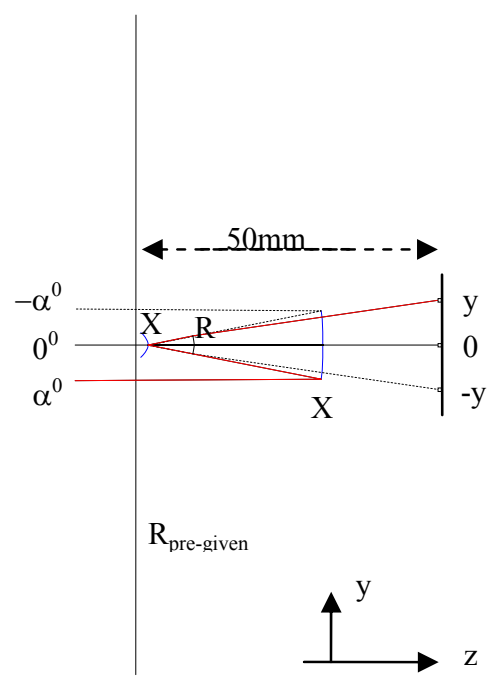

Solution 2

Fig 4. Initial curves selection in tele-objective design

The front surface is a parabola with very small central curvature

Then by using the SMS2D growth algorithm for the two selections of initial curves, two RXXR designs and their ray-tracing results for meridian ray-bundles are obtained, as shown in Fig 5. Comparing these two ray-tracing results, it can be seen that solution 1 has better control of meridian rays than solution 2 .

In the design procedure described above, the first refractive surface is pre-given a certain curvature. This affords an additional freedom to optimize the optical system, because we have only designed the system with meridian rays, without controlling skew rays. The optimization is achieved by simply varying the central curvature of the first given parabola, and then continuing the SMS2D construction for the rest three curves, which is a one-variable optimization process. The merit function for this optimization is the largest RMS spot size, calculated by ray-tracing both meridian rays and skew rays. The results of the optimization are shown in Fig 6. It can be seen that varying the central curvature of the first parabola doesn't significantly change the RMS spot size for meridian rays(RMS2D). However, the RMS spot size for both meridian rays and skew rays(RMS3D) does drop from $82 \mathrm{um}$ to $15 \mathrm{um}$. The reason why SMS is so effective in imaging design could be that: First, by controlling the on-axis meridian rays, spherical aberration is removed; Also, by optimization the central curvature of the first parabola, astigmatic aberration is also minimized. Analysis in Code V without optimization inside Code V, shown in Fig 7, also confirms the explanation that only a small coma type aberration appears in the optical system. 


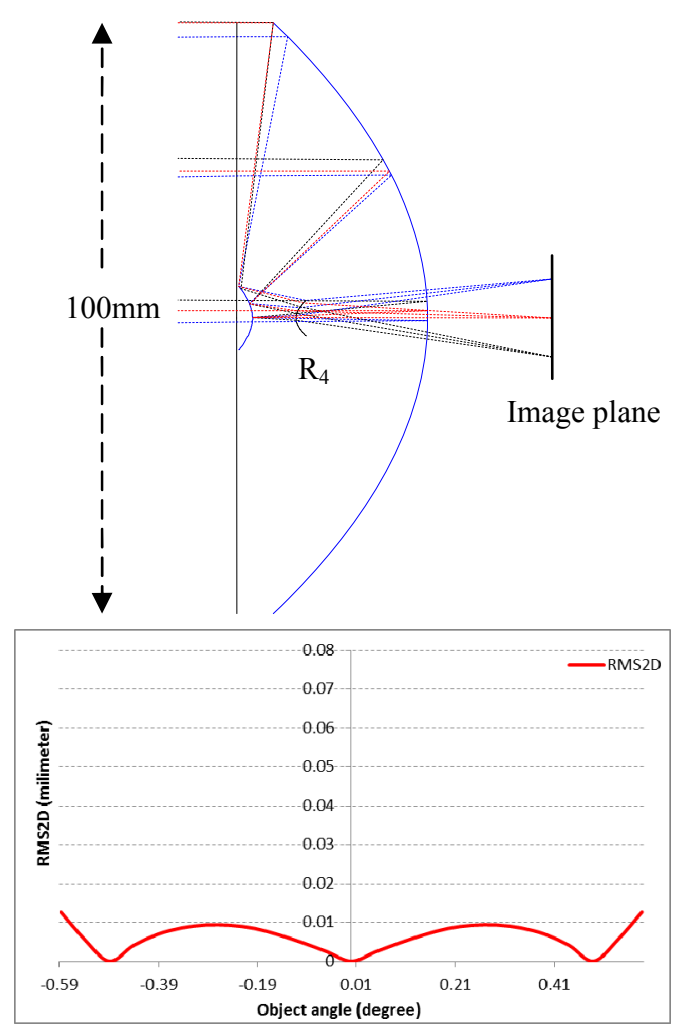

a)

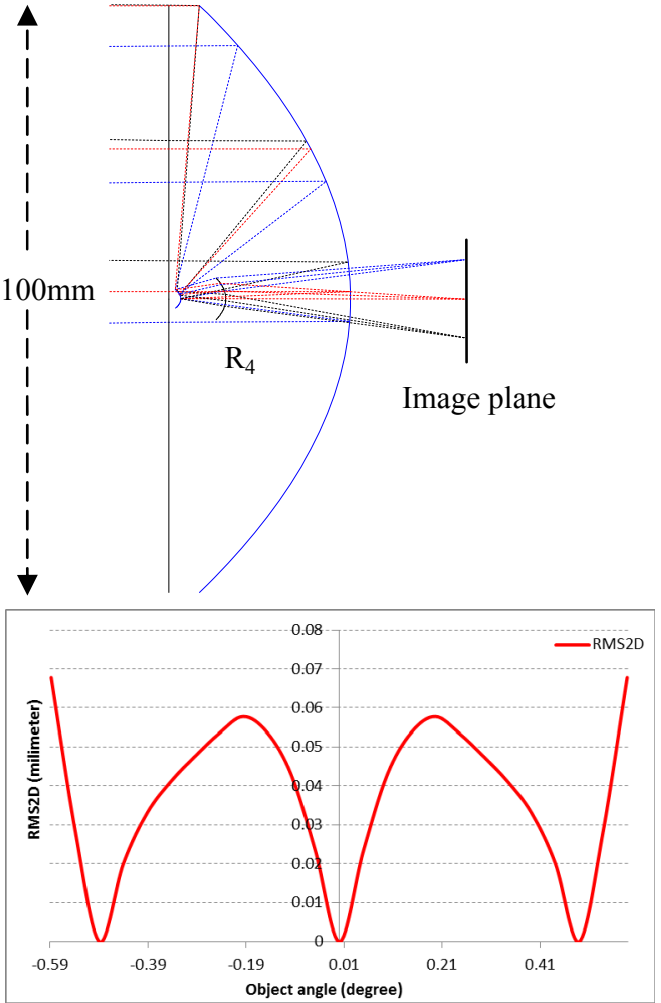

b)

Fig 5. SMS construction for 2 candidate selections of initial curves:

a) With one concave type refractive exit surface $\mathrm{R}_{4}, \mathrm{RMS} 2 \mathrm{D}<12 \mu \mathrm{m}$

b) With one convex type refractive exit surface $\mathrm{R}_{4}, \mathrm{RMS} 2 \mathrm{D}<70 \mu \mathrm{m}$

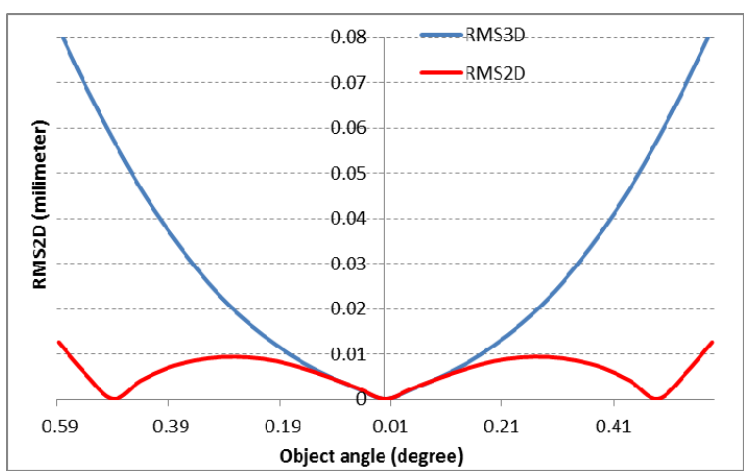

a. before optimization, central curvature $=0$

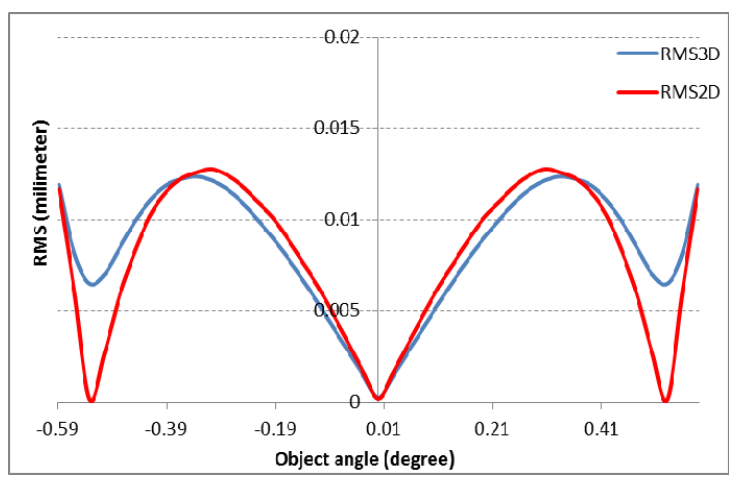

b. after optimization, central curvature $=0.00055(1 / \mathrm{mm})$

Fig 6. SMS Optimization results of central curvature of first parabola

a) Stigmatic aberrations is observed over all fields of view

b) Stigmatic aberrations is reduced significantly over all fields of view 

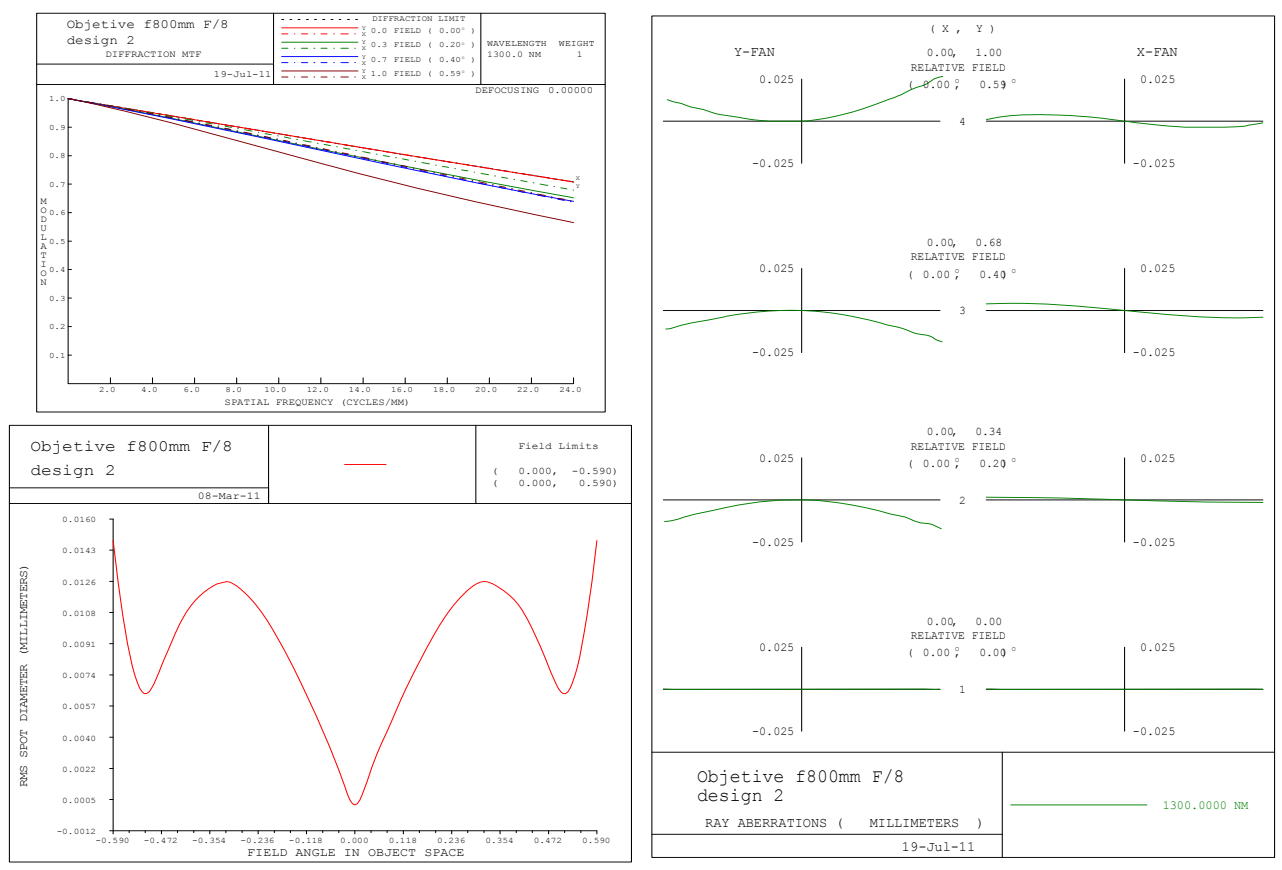

Fig 7. Code $\mathrm{V}$ analysis results for RXXR design:

MTF, RMS3D spot size and Ray aberration curves

Code V results for the XRXR type design are shown in Fig 8.
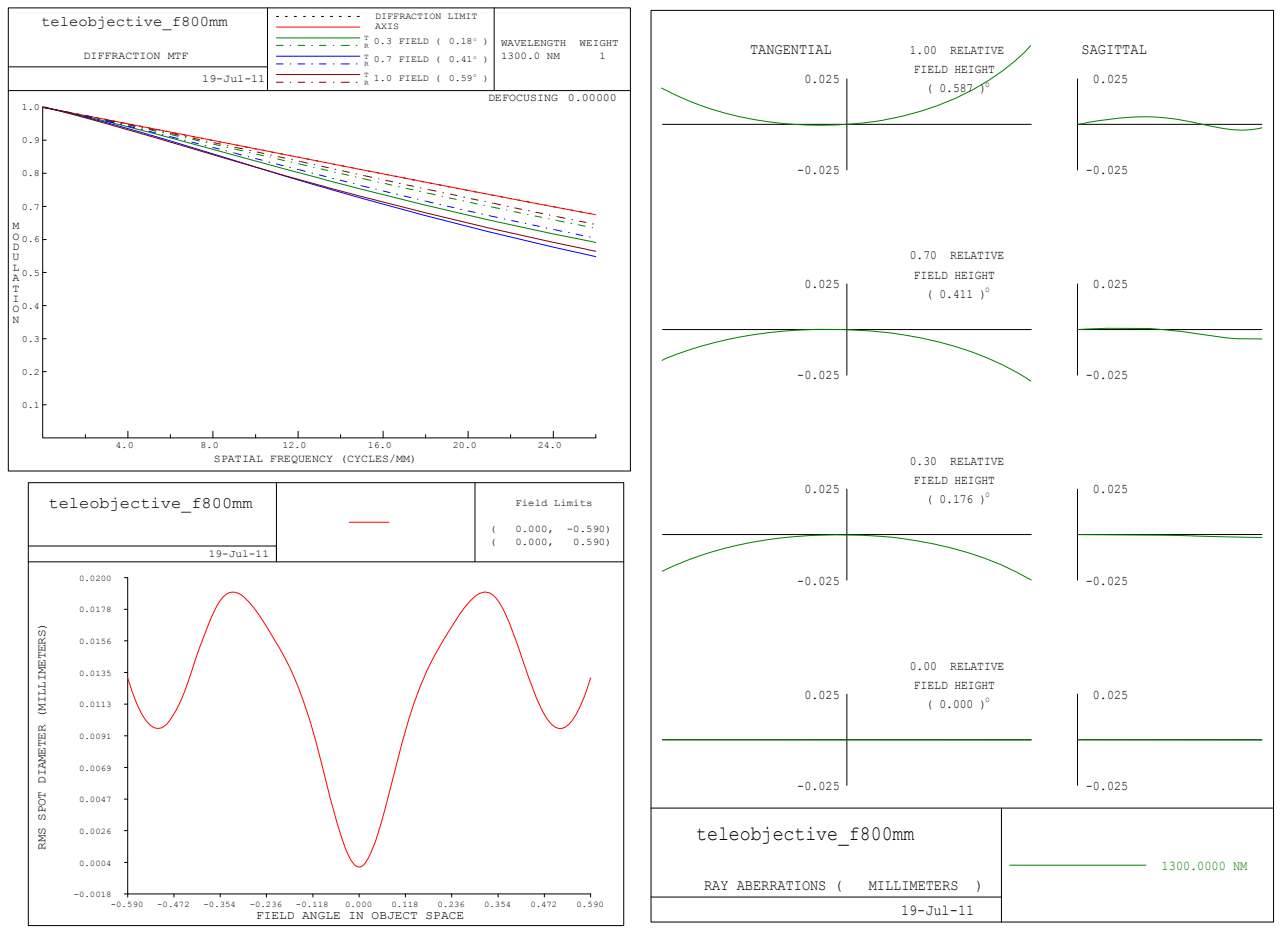

Fig 8. Code V analysis results for XRXR design:

MTF, RMS3D spot size and Ray aberration curves 
Finally, obtained monochromatic designs are used as initial designs for further optimization with real materials inside Code V. Compared to the visible band, the SWIR band is much wider, almost twice as length. Using XRXR design as a starting point however can lead to a very simple achromatic strategy by adding one small lens after central big lens. Fig 9 shows optimization results for a XRXR type design with overall system length of less than $60 \mathrm{~mm}$. Chromatic aberration has been corrected by an addition small lens with material NKF9. Distortion of the system results in less than $2.5 \%$. The simplicity of this final configuration demonstrates that a good starting point can not only make design process more efficient, but also reduce the complexity of optical system design.

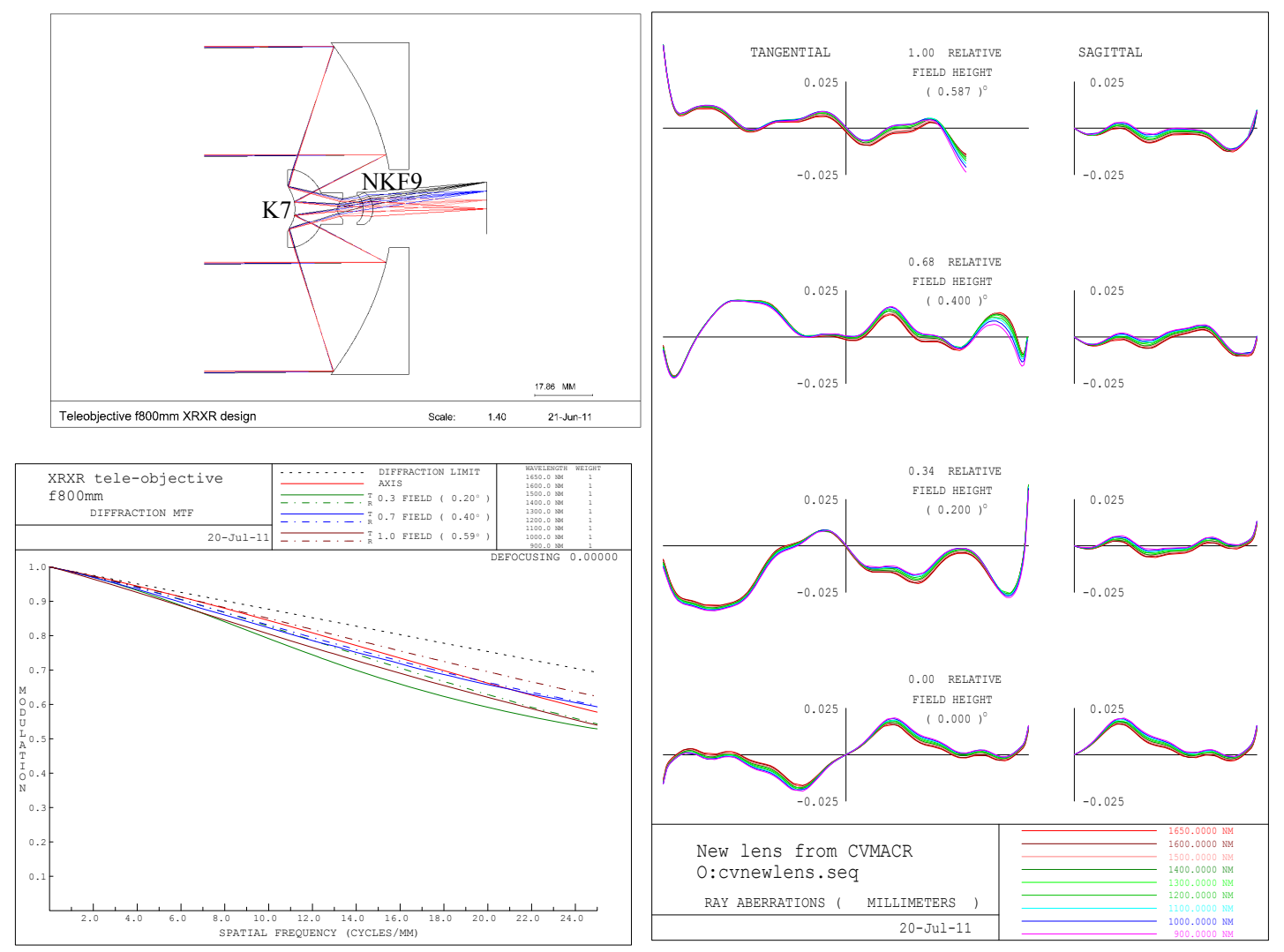

Fig 9. XRXR achromatic design in Code V:

MTF and Ray aberration curves, distortion $<2.5 \%$

\section{Conclusion}

In this work, a set of wavefront criteria was proposed for the selection of SMS initial curves. Solutions to the wavefront criteria can help designers explore possible central shapes of the optical system, which will lead to different optical surfaces after SMS construction. Designed rays are then traced through all of these solutions to determine the best one.

In monochromatic tele-objective design, the SMS construction method is implemented with a single prescribed surface. It has been seen that control over the designed meridian rays doesn't change a lot with different prescribed surfaces. Therefore, the additional freedom given by the prescribed surface can be utilized to control skew rays. By only optimizing one variable, which is the central curvature of the prescribed parabola, the astigmatic aberration 
is minimized from $80 \mathrm{um}$ to $15 \mathrm{um}$. Code $\mathrm{V}$ analysis further shows that only a small coma aberration remains in the optical system.

By using XRXR type SMS design as initial point for Code V optimization, an ultra-compact and high performance tele-objective design is found with common glasses.

\section{Acknowledgement}

Authors thank the Spanish Ministries MCINN (ENGINEERING METAMATERIALS: CSD2008-00066, DEFFIO: TEC2008-03773, SIGMASOLES: PSS-440000-2009-30), MITYC (ECOLUX: TSI-020100-2010-1131, SEM: TSI-020302-2010-65) and the Madrid Regional Government (SPIR: 50/2010O.23/12/09,TIC2010 and O-PRO: PIE/209/2010) and UPM (Q090935C59) for the support given in the preparation of the present work. The authors thank Synopsys (formerly Optical Research Associates) for granting us the CodeV university license

\section{Reference}

[1] D. Action, et. al., "Large Format Short Wave Infrared (SWIR) Focal Plane Array (FPA) With Extremely Low Noise and High Dynamic Range," Proc. SPIE 7298, pp. 7298-1 - 13 (2009).

[2] Marc P. Hansen and Douglas S. Malchow, "Overview of SWIR detectors, cameras, and applications", Proc. SPIE 6939, 69390I (2008).

[3] J. Battaglia, et. al., "An uncooled 1280 x 1024 InGaAs focal plane array for small platform, shortwave infrared imaging," Proc. SPIE 7298, pp. 72983C-1 - 8 (2009).

[4] R. Hamilton Shepard III and Scott Sparold, "Material Selection for Color Correction in the Short-wave Infrared," Proc. SPIE 7060, pp. 70600E-1 - 10 (2008).

[5] Craig Olson, Tim Goodman, Chris Addiego and Steve Mifsud, "Design and construction of a short-wave infrared 3.3X continuous zoom lens", Proc. SPIE 7652, 76522A (2010).

[6] Chapter 8 in R.Winston, J.C. Miñano, P. P. Benítez, with contributions of N. Shatz, J. Bortz, "Nonimaging Optics", Academic Press Elsevier (2004).

[7] Chaves, J., [Introduction to Nonimaging Optics], CRC Press, (2008).

[8] Pablo Benítez, Juan C. Miñano, José Blen, Rubén Mohedano, Júlio Chaves, Oliver Dross, Maikel Hernández, and Waqidi Falicoff, "Simultaneous multiple surface optical design method in three dimensions," Opt. Eng. 43, 1489 (2004).

[9] P. Benítez and J. C. Miñano, "Ultra high-numerical-aperture imaging concentrator," J. Opt. Soc. Am. A, Vol 14 (1997).

[10] J. C. Miñano, P. Benítez, Wang Lin, José Infante, Fernando Muñoz, Asunción Santamaría

"An application of the SMS method for imaging designs," Optical Express, Vol 17, No. 26, p. 24036 (2009).

[11] Miñano, J.C., Benítez, P., Wang Lin,, Muñoz, F., Infante, J., Santamaría, A., "Overview of the SMS design method applied to imaging optics", Proc. SPIE 7429, 74290C (2009).

[12] Jose M. Infante Herrero, Fernando Muñoz, Pablo Benitez, Juan Carlos Miñano, Wang Lin, Juan Vilaplana, Guillermo Biot, Marta de la Fuente "Novel fast catadioptric objective with wide field of view", Proc. SPIE 7787, 778704 (2010).

[13] F. Muñoz, P. Benítez, and J. C. Miñano "High-order aspherics: the SMS nonimaging design method applied to imaging optics”, Proc. SPIE 7061, 70610G (2008). 
[14] Fernando Muñoz Doctoral Disertation "Sistemas ópticos avanzados de gran compactibiliad con aplicaciones en formación de imagen y en iluminación" UPM, Madrid (2004)

[15] Orestes N. Stavroudis, “The mathematics of geometrical and physical optics", Wiley-VCH (2006). 